\title{
Nanostructured Transparent Conductive Oxide Films for Plasmonic Applications
}

Kim, Jongbum; Zhao, Yang; Naik, Gururaj V.; Emani, Naresh K.; Guler, Urcan; Kildishev, Alexander V.; Alu, Andrea; Boltasseva, Alexandra

Published in:

Proceedings of 2013 Conference on Lasers and Electro-Optics (CLEO)

Publication date:

2013

Document Version

Publisher's PDF, also known as Version of record

Link back to DTU Orbit

Citation (APA):

Kim, J., Zhao, Y., Naik, G. V., Emani, N. K., Guler, U., Kildishev, A. V., Alu, A., \& Boltasseva, A. (2013). Nanostructured Transparent Conductive Oxide Films for Plasmonic Applications. In Proceedings of 2013 Conference on Lasers and Electro-Optics (CLEO) [QTh3B.8] IEEE.

\section{General rights}

Copyright and moral rights for the publications made accessible in the public portal are retained by the authors and/or other copyright owners and it is a condition of accessing publications that users recognise and abide by the legal requirements associated with these rights.

- Users may download and print one copy of any publication from the public portal for the purpose of private study or research.

- You may not further distribute the material or use it for any profit-making activity or commercial gain

- You may freely distribute the URL identifying the publication in the public portal 


\title{
Nanostructured Transparent Conductive Oxide Films for Plasmonic Applications
}

\author{
Jongbum Kim ${ }^{1}$, Yang Zhao ${ }^{2}$, Gururaj V. Naik ${ }^{1}$, Naresh K. Emani ${ }^{1}$, Urcan Guler ${ }^{1}$, \\ Alexander V. Kildishev ${ }^{1}$, Andrea Alu ${ }^{2}$ and Alexandra Boltasseva*,1,3,4 \\ ${ }^{1}$ School of Electrical and Computer Engineering and Birck Nanotechnology Center, Purdue University, West Lafayette, IN 47907, USA \\ ${ }^{2}$ Department of Electrical and Computer Engineering, The University of Texas at Austin, 1 University Station C0803, Austin, TX 78712, USA \\ ${ }^{3}$ DTU Fotonik, Department of Photonics Engineering, Technical University of Denmark, Lyngby, DK-2800, Denmark \\ ${ }^{4}$ Erlangen Graduate School of Advanced Optical Technologies (SAOT), Universität Erlangen-Nürnberg, 91052 Erlangen, Germany \\ Email: aeb@purdue.edu*
}

\begin{abstract}
Transparent conductive oxides (TCOs) as substitutes to metals could offer many advantages for low-loss plasmonic and metamaterial (MM) applications in the near infrared (NIR) regime. By employing a lift-off process, we fabricated 2D-periodic arrays of TCO nanodisks and characterized the material's plasmonic properties to evaluate the performance of TCOs as metal substitutes.

OCIS codes: (160.4670) Optical materials; (250.5403) Plasmonics; (160.3918) Metamaterials;
\end{abstract}

Recent studies in searching for better plasmonic materials demonstrate that TCOs are good alternatives to noble metals in the NIR because they exhibit metallic behavior and have smaller material loss compared to those of silver and gold in the NIR regime [1-4]. For the experimental realizations of optical devices, in which conventional metals are replaced by new materials, the next important step is to develop nanopatterning techniques which enable the new classes of optical devices in the NIR. This is an essential study because typical plasmonic and MM devices are built with nanostructured metals and dielectrics as their building blocks. Furthermore, the performance of nano-patterned TCO thin films should be evaluated to verify that their optical properties can hold under the various required fabrication processes. In this paper, we investigate the lift-off technique for TCOs with electron-beam lithography (EBL) and fabricated 2D-periodic arrays of nanodisks with gallium-doped zinc oxide (GZO) and characterized the material's plasmonic properties. We also showed the effect of disk size on the plasmonic performance and the tunability of plasmonic properties of GZO nanodisk by varying the amount of dopant while maintaining the shape and size of the nanodisks.

TCO thin films such as aluminum doped zinc oxide (AZO), GZO, tin doped indium oxide (ITO) are prepared by pulsed laser deposition under low deposition temperature $\left(70^{\circ} \mathrm{C}\right)$ to avoid e-beam resist $(\mathrm{ZEP})$ being baked out. In Fig. $1 \mathrm{~b}$ and $1 \mathrm{c}$, the optical properties of these materials are plotted. Notably, the cross-over wavelengths of all the TCO films are below telecommunication wavelength of $1.55 \mu \mathrm{m}$. The AZO film offers the lowest optical loss, referring to the imaginary part of its permittivity and GZO can provide a cross-over wavelength as low as $1.2 \mu \mathrm{m}$, but the optical loss in GZO is higher than that in AZO. Under deposition conditions optimized for the lift-off process, the ITO film has the highest loss and the largest cross-over wavelength compared to AZO and GZO. Since GZO exhibits the strongest metallic behavior compared to AZO and ITO, we fabricated the 2D nanodisk arrays with GZO material. To fabricate a 2D array of GZO nanodisks, a silicon substrate was first spin-coated with a 1- $\mu$ m-thick layer of ZEP and then the pattern of nanodisk array is exposed by electron beam. The exposed sample was developed in ZED-N50 and GZO film is deposited on the patterned silicon wafer. Lastly, sample deposited with GZOs is dipped in ZDMAC to lift-off the ZEP. The scanning electron microscope (SEM) image in Fig. 1a shows the nano-patterned GZO nanodisk arrays with $250 \mathrm{~nm}$ disk diameter and $270 \mathrm{~nm}$ heights.

The measurement of transmission spectra was performed with FTIR in the wavelength ranges from $1.25 \mu \mathrm{m}$ to 10 $\mu \mathrm{m}$. From the FTIR measurement in Fig 1d, we can clearly observe well-defined localized surface plasmon resonance (LSPR) peaks and the position of the peaks is well matched by simulation results. We also investigated the effects of disk size and doping density on the (LSPR) properties. In this experiment, we measured the transmission spectra with spectroscopic ellipsometer in the wavelength range from $1.1 \mu \mathrm{m}$ to $2.4 \mu \mathrm{m}$ in order to accurately detect the variation of LSPR peak. The positions of these peaks depend on both the disk size and the doping density. As the disk diameter increases (see fig. 1e), the disk arrays begin to support higher order plasmonic modes that start to overlap, hence the resonance peak becomes broader and stronger. In addition, as the doping 
density of GZO increases (see Fig. 1f), the films exhibit higher plasma frequency, thus the resonance shifts to shorter wavelengths. The dependence of optical properties of GZO on doping density has been investigated in our previous study [2]. In terms of the tunability of the LSPR wavelength, the peak shift arising from the change in doping density is much stronger than that caused by the nanodisk geometry. Fig. $1 \mathrm{~g}$ shows the simulation results with Finite Element Method (FEM) based COMSOL Multiphysics for the GZO nanodisks with varying doping densities. Although some broadening of the experimental spectra due to fabrication imperfections and change of cyrstallinity and grain size during fabrication exists, the positions of resonance features are in good agreement with the experiments. [5, 6]
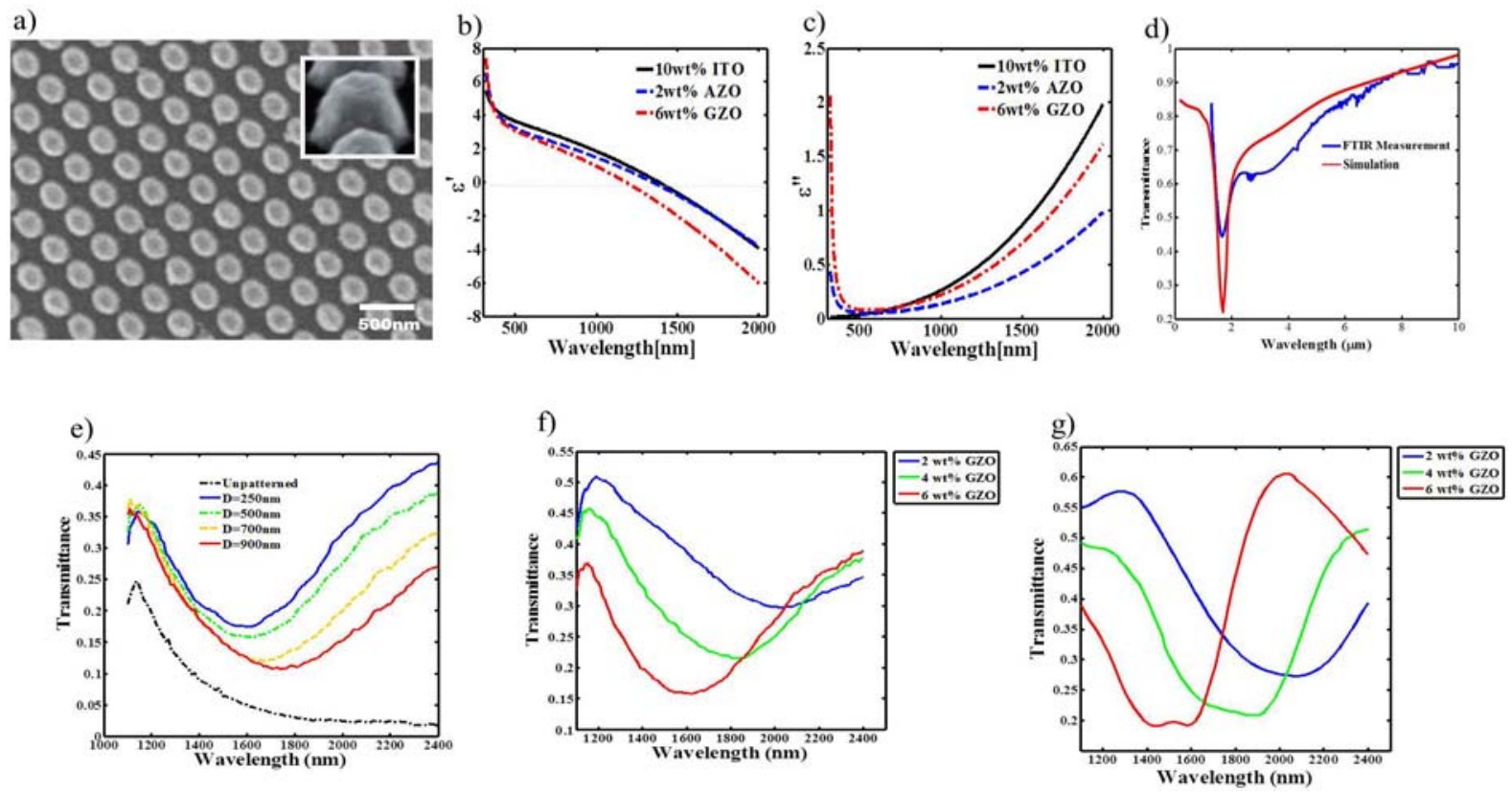

Fig 1. a) SEM image of top-down view of the nanodisks (inset) $54^{0}$ tilted SEM image of an array of GZO nanodisks. Optical properties of TCO thin films: b) real and c) imaginary parts of permittivity of AZO, GZO and ITO films extracted from ellipsometry measurements. d) Transmittance spectra for GZO nanodisk array by FTIR measurement and the FEM simulation results of transmittance spectra. e) Transmittance spectra for GZO nanodisk array samples and unpatterned GZO thin film on Si substrates with different nanodisk diameters. f) Measured transmittance spectra for the GZO nanodisk arrays (disk diameter of $500 \mathrm{~nm}$ ) with different doping ratios in the GZO material. g) Simulation results of transmittance spectra for GZO nanodisk arrays using different dielectric functions for films with different doping concentrations.

In conclusion, TCOs appear to be good alternatives to noble metals for plasmonic applications in the NIR and TCOs are also advantageous because standard nanofabrication techniques can be employed to make nanostructured samples. When patterned, these materials exhibit LSPR properties similar to gold and silver nanostructures. In contrast to noble metals, the resonance properties strongly depend on the properties of the film such as carrier concentration. Such tunable properties of TCO could be used to tailor the optical resonances for various plasmonic applications and enable a new generation of controllable, switchable devices.

\section{References}

P. R. West, S. Ishii, G. V. Naik, N. K. Emani, V. M. Shalaev, and A. Boltasseva, "Searching for better plasmonic materials," Laser \& Photonics Reviews, vol. 4, pp. 795-808, 2010.

G. V. Naik, J. Kim, and A. Boltasseva, "Oxides and nitrides as alternative plasmonic materials in the optical range," Optical Materials Express, vol. 1, pp. 1090-1099, 2011.

G. V. Naik, J. Liu, A. V. Kildishev, V. M. Shalaev, and A. Boltasseva, "Demonstration of Al: ZnO as a plasmonic component for near-infrared metamaterials," Proceedings of the National Academy of Sciences, vol. 109, pp. 8834-8838, 2012. A. Boltasseva and H. A. Atwater, "Low-loss plasmonic metamaterials," Science, vol. 331, p. 290, 2011.

[5] K. P. Chen, V. P. Drachev, J. D. Borneman, A. V. Kildishev, and V. M. Shalaev, "Drude relaxation rate in grained gold nanoantennas," Nano Letters, vol. 10, pp. 916-922, 2010.

[6] V. P. Drachev, U. K. Chettiar, A. V. Kildishev, H. K. Yuan, W. Cai, and V. M. Shalaev, "The Ag dielectric function in plasmonic metamaterials," Optics Express, vol. 16, pp. 1186-1195, 2008. 